\title{
Enquêtes nord-américaines sur les violences homophobes et transphobes en contexte scolaire : l'invisibilisation du sexisme
}

\author{
Line Chamberland
}

\begin{abstract}
Résumé
Cet article propose une lecture critique de trois enquêtes réalisées respectivement au Canada anglais, au Québec et aux États-Unis, sur le climat scolaire et les violences interpersonnelles entre pairs fondées sur la non-conformité aux normes sexuelles et de genre, dans le milieu scolaire. Il relève l'ampleur de ces violences, qui peuvent toucher tous les élèves, ainsi que la victimisation relativement plus importante des jeunes trans ou non cisgenres et des élèves dont l'expression de genre est non conforme aux normes de genre. Toutefois, ces enquêtes mettent au second plan l'analyse des violences sexistes ou subies en tant que filles, y compris par les filles non hétérosexuelles, de même que l'examen des auteur·e's des violences interpersonnelles. Tout en constatant l'enchevêtrement et le caractère systémique des violences liées à la sexualité et au genre, ces études négligent de considérer l'asymétrie entre filles et garçons et le rôle des violences interpersonnelles dans la reproduction des rapports sociaux de sexe.

VIOLENCES INTERPERSONNELLES - ORIENTATION SEXUELLE - SEXISME HOMOPHOBIE - TRANSPHOBIE - IDENTITE DE GENRE - EXPRESSION DE GENRE AMERIQUE DU NORD

Depuis environ une dizaine d'années, au Canada et aux ÉtatsUnis, le phénomène de l'intimidation en contexte scolaire a fait l'objet d'une importante médiatisation, souvent déclenchée par
\end{abstract}


des cas tragiques de suicides d'adolescent $\cdot e \cdot s$, dont des jeunes présumés gais (Petit et al., 2011). Diverses recherches nordaméricaines se sont penchées sur le phénomène. Certaines études ont examiné spécifiquement les violences exercées par des pairs envers d'autres élèves en raison de l'orientation homoou bisexuelle qui leur est attribuée, à tort ou à raison, ou de leur non-conformité aux normes de genre, des violences respectivement qualifiées d'homophobes et de transphobes. Ces enquêtes interrogent tantôt l'ensemble des jeunes, tantôt celles et ceux qui s'identifient comme lesbiennes, gais, bisexuel-le·s, transsexuel·le's, queer, en questionnement (LGBTQ) ou avec d'autres termes signifiant qu'ils ne sont pas exclusivement hétérosexuel·le·s ou cisgenres ${ }^{1}$. Elles forment déjà un corpus intéressant pour examiner les représentations scientifiques - et sociales - du genre et des violences de genre. Elles n'accordent pas une large place aux concepts théoriques, mais elles construisent et véhiculent un certain point de vue sur ces phénomènes.

Cet article propose une lecture critique de la notion de genre telle qu'elle s'installe et se met en œuvre dans un échantillon de ces études. Les trois études retenues ont avant tout été choisies pour l'envergure de leur diffusion et donc de leur rayonnement. Ces études proposent une certaine compréhension des violences fondées sur le genre à travers des choix méthodologiques qu'elles ont fait, notamment dans la conception du questionnaire et l'analyse des résultats d'enquête. Mon intention ici n'est pas d'élaborer une critique exhaustive de l'ensemble de leur méthodologie, mais de relever les aspects de leur «opérationnalisation» qui conduisent à certaines représentations de ce qui constitue des « violences de genre». La poursuite de cette compréhension m'animait au moment où je menais moi-même l'une de ces études, laquelle fait partie de

\footnotetext{
${ }^{1}$ Le terme «cisgenre » a émergé des discours activistes trans des années 1990. Il désigne une personne qui ne s'identifie pas comme transgenre ou dont l'identité de genre correspond au sexe qui lui a été assigné à la naissance (Aultman, 2014). Les termes d'autodénomination des identités sexuelles et de genre se multiplient, parmi lesquels plusieurs expriment un refus de la bicatégorisation de ces identités, comme genderqueer, non binaire ou pansexuel (Veale et al., 2015).
} 
l'échantillon retenu pour cette raison. Les questions que je pose aux trois études sélectionnées sont les mêmes et m'ont permis une sérieuse autocritique de la recherche que j'ai moi-même dirigée. Ces questions sont les suivantes :

- Quels éclairages sont projetés sur les violences fondées sur le genre et quels aspects sont laissés dans l'ombre?

- Comment s'articulent, ou non, les violences sexistes et les violences homophobes et transphobes?

- Quelle place est accordée aux violences lesbophobes dans les analyses?

- Quels sont les impensés théoriques et les angles morts qui en résultent sur le plan empirique?

Après avoir présenté les études sélectionnées, j'examinerai, pour chacune d'elle, le traitement empirique et conceptuel des observations portant sur les violences fondées sur le genre, afin d'en saisir les particularités et les limites puis je conclurai sur leur commune oblitération, du moins partielle, des violences hétéronormatives exercées sur les filles.

\section{Les études sélectionnées}

Trois rapports d'enquête (Canada, Québec, États-Unis) ont donc été sélectionnés en raison de leur potentiel illustratif des apports et des écueils de ce type de recherches pour documenter les violences fondées sur le genre et leur articulation avec les violences liées à l'orientation sexuelle. Ils présentent les résultats d'études portant sur le climat scolaire par rapport à la diversité sexuelle et de genre, et examinent entre autres les propos dénigrants et les expériences de victimisation rapportées par des élèves dans un contexte scolaire. Ils examinent également les réponses des établissements face aux violences interpersonnelles à caractère homophobe ou transphobe. Largement diffusés en vue de susciter l'adoption de politiques et de mesures de prévention des violences scolaires, ils ont bénéficié, dans leur cadre national respectif, d'un rayonnement 
notable au sein de la communauté scientifique, des milieux de l'éducation et des milieux associatifs LGBTQ.

Le tableau suivant situe ces trois études qui se caractérisent par leur visée descriptive, leur recours à de larges échantillons, représentatifs ou non, leur caractère empirique et leur faible niveau de théorisation. Les enquêtes canadienne et québécoise se sont déroulées dans la même période et une entente avait été conclue entre les deux équipes de recherche pour délimiter le territoire de la collecte de données de chaque enquête, afin d'éviter tout chevauchement dans le recrutement. Aux ÉtatsUnis, l'organisme Gay, Lesbian, Straight Education Network (GLSEN) mène des enquêtes bisannuelles sur les violences en milieu scolaire depuis 1999 en utilisant un questionnaire évolutif, afin entre autres d'intégrer la diversification croissante des termes d'autodésignation chez les jeunes LGBTQ. C'est l'édition 2013 qui a été retenue ici. Il faut préciser que les autres enquêtes canadienne et québécoise se sont largement inspirées de l'outil d'enquête de GLSEN dans la version qui était alors disponible (édition 2005).

Sauf indications contraires, la critique proposée dans cet article porte sur les rapports d'enquête diffusés auprès d'un large public. Puisque la visée ici n'est pas de faire un portrait empirique $^{2}$ ni une critique méthodologique approfondie, les données statistiques seront généralement omises ou fournies uniquement à titre indicatif. D'autres précisions s'imposent d'emblée. Aucune de ces études ne se revendique explicitement comme féministe. Le concept de « genre » n'est pas défini dans ces rapports d'enquêtes et la mention du genre renvoie à des caractéristiques identitaires individuelles, sans référence aux « rapports sociaux de genre ». Les notions d'homophobie et de transphobie sont mises en avant parallèlement, afin de distinguer - avec peu de succès comme le montrera la suite de l'article - les violences suscitées par un écart aux normes sexuelles vs un écart aux normes de genre. Le terme homophobie englobe alors la gaiphobie, la lesbophobie - une notion quasiment absente - et la biphobie, cette dernière étant

\footnotetext{
${ }^{2}$ Pour des métasynthèses récentes sur les violences LGBT-phobes et leurs impacts, voir Toomey et Russell (2016) et Fedewa et Ahn (2011).
} 
parfois mentionnée afin de souligner l'existence de préjugés spécifiques envers la bisexualité, y compris dans les milieux LGBTQ. Pour sa part, le rapport de l'enquête québécoise ne traite pas des violences transphobes, qui ont été examinées à partir d'une méthodologie qualitative dans un rapport distinct (Chamberland, Baril et Duchesne, 2011).

\section{Étude 1 - Every Class in Every School : le sexisme escamoté}

Le rapport Every Class in Every School (Taylor et al., 2011) présente les résultats d'un sondage en ligne auprès d'un échantillon constitué à l'aide de deux processus parallèles de recrutement :

a) l'accès à un sondage sur des ordinateurs scolaires par des groupes d'élèves fréquentant des écoles réparties à travers le Canada (sauf le Québec);

b) l'appel à des volontaires joints via des associations LGBTQ, principalement celles s'adressant aux jeunes. Au final, les élèves LGBTQ sont surreprésentés, avec $26 \%$ des répondant $\cdot \mathrm{e} \cdot \mathrm{s}$ s'identifiant comme lesbiennes, gais, bisexuel·le's, queer ou en questionnement, et $3 \%$ comme trans (transsexuel $\cdot$ le ou transgenre) ou bispirituel $\cdot 1 \mathrm{e}^{3}$.

\footnotetext{
${ }^{3}$ La bispiritualité est un concept des Premières nations qui indique à la fois une identité autochtone et une appartenance à la diversité sexuelle et de genre. Elle comporte aussi une dimension spirituelle qui varie selon les individus et selon les communautés autochtones (Dubuc, 2017, p. 9).
} 
Les études sélectionnées en résumé

\begin{tabular}{|c|c|c|c|c|c|}
\hline $\begin{array}{l}\text { Référence du rapport } \\
\text { d'enquête }\end{array}$ & $\begin{array}{l}\text { Lieu de } \\
\text { l'enquê } \\
\text { te }\end{array}$ & $\begin{array}{l}\text { Partenair } \\
\text { es de } \\
\text { recherche }\end{array}$ & $\begin{array}{l}\text { Collecte des } \\
\text { données }\end{array}$ & $\begin{array}{l}\text { Nombre et âge des } \\
\text { répondant·e·s }\end{array}$ & $\begin{array}{l}\text { Auto-identification } \\
\text { des répondant·e·s }\end{array}$ \\
\hline $\begin{array}{l}\text { Every class in every } \\
\text { school, Taylor et coll., } \\
2011\end{array}$ & $\begin{array}{l}\text { Canada } \\
\text { (sauf } \\
\text { Québe } \\
\text { c) }\end{array}$ & $\begin{array}{l}\text { Égale } \\
\text { Canada* }\end{array}$ & $\begin{array}{l}\text { 2007-2009 } \\
\text { Questionnaire } \\
\text { en classe et } \\
\text { en ligne }\end{array}$ & $\begin{array}{l}\mathrm{N}=3607 \\
92 \% \text { entre } 12-17 \text { ans } \\
\text { Classes sélectionnées } \\
\text { et individus } \\
\text { volontaires }\end{array}$ & $\begin{array}{l}\text { Mixte dont } \\
26 \% \text { lesbienne, gai, } \\
\text { bi, queer, } \\
\text { bispirituel.le } \\
3 \% \text { trans, } \\
\text { bispirituel·le }\end{array}$ \\
\hline $\begin{array}{l}\text { L'homophobie à } \\
\text { l'école secondaire au } \\
\text { Québec. Portrait de la } \\
\text { situation, impacts et } \\
\text { pistes de solution, } \\
\text { Chamberland et al., } \\
2011\end{array}$ & $\begin{array}{l}\text { Québe } \\
c\end{array}$ & $\begin{array}{l}\text { Réseau } \\
\text { de } \\
\text { partenair } \\
\text { es** }\end{array}$ & $\begin{array}{l}2009 \\
\text { Questionnaire } \\
\text { en classe }\end{array}$ & $\begin{array}{l}\mathrm{N}=2747 \\
14-17 \text { ans } \\
\text { Échantillon } \\
\text { représentatif d'élèves }\end{array}$ & $\begin{array}{l}\text { Mixte dont } \\
8 \% \text { lesbienne, gai, } \\
\text { bi, queer, en } \\
\text { questionnement } \\
\text { (Pas de question sur } \\
\text { l'identité de genre) }\end{array}$ \\
\hline $\begin{array}{l}\text { The } 2013 \text { National } \\
\text { School Climate Survey, } \\
\text { Kosciw et al., } 2014\end{array}$ & $\begin{array}{l}\text { États- } \\
\text { Unis }\end{array}$ & GLSEN*** & $\begin{array}{l}2013 \\
\text { Questionnaire } \\
\text { en ligne et } \\
\text { format papier }\end{array}$ & $\begin{array}{l}\mathrm{N}=7898 \\
13-21 \text { ans } \\
\text { Individus volontaires }\end{array}$ & $\begin{array}{l}\text { Diverses identités } \\
\text { non exclusivement } \\
\text { hétérosexuelles ou } \\
\text { non cisgenres }\end{array}$ \\
\hline
\end{tabular}

* Organisme canadien de défense des droits des personnes lesbiennes, gaies, bisexuelles, transsexuelles, queer, intersexes et bispirituelles (LGNTQI2S) (www.egale.ca)

** Ministère de l'Éducation, directions d'établissements, syndicats du personnel enseignant et autre, organismes associatifs intervenant en milieu scolaire. Ces organismes sont réunis dans la Table nationale de lutte contre l'homophobie et la transphobie dans les réseaux scolaire et collégial qui existe depuis 2005 (http://www.colloquehomophobie.org/table-nationale)

*** Organisme états-unien de recherche et de défense des droits des personnes LGBTQ dont la mission est de rendre les écoles sécuritaires et conviviales pour tous les élèves, indépendamment de leur orientation sexuelle, de leur identité ou de leur expression de genre ${ }^{4}$ (www.glsen.org).

\footnotetext{
${ }^{4}$ www.glsen.org
} 


\section{L'assimilation conceptuelle du sexisme et de la transphobie}

L'étude s'est entre autres intéressée aux propos homophobes entendus dans le contexte scolaire, soit les commentaires dévalorisants (It's so gay!) et les insultes telles que traiter un autre élève de faggot, queer, dyke ou lesbo. Les deux types de propos sont rapportés comme étant «très» ou «assez fréquents », alors que seule une minorité d'élèves indique ne les entendre que « rarement » ou « jamais ». Une section du rapport traite des commentaires négatifs relativement au genre en distinguant les réactions négatives face à la non-conformité de genre et les propos dénigrant l'un ou l'autre sexe en général. Les premiers cibleraient plus souvent les garçons qui ne se montrent pas assez virils, comparativement aux filles qui ne seraient pas assez féminines. Autrement dit, les garçons se verraient plus souvent reprocher leur manque de masculinité que les filles, leur manque de féminité. Les réactions négatives à ces manquements sont qualifiées de transphobes par les auteur'e's de l'enquête, sans plus d'explication, plutôt que de sexistes ou d'homophobes (en référence aux stéréotypes de l'homosexuel efféminé et de la lesbienne masculine). Le qualificatif de «sexiste» est réservé aux commentaires dénigrant l'un ou l'autre sexe en général. À cet égard, la situation serait inversée, c'est-à-dire que de tels propos seraient beaucoup plus fréquents à propos des filles que des garçons; en d'autres termes, le sexe féminin est plus souvent dénigré que le sexe masculin. Les auteur $\cdot e \cdot s$ notent que les répondant $\cdot e \cdot s$ trans sont proportionnellement plus nombreux et nombreuses à rapporter ces deux types de commentaires, comparativement aux non-trans, et suggèrent deux pistes pour expliquer cette différence : d'une part, la plus grande sensibilité des élèves trans aux remarques entendues dans leur entourage relativement au genre et, d'autre part, la possibilité que de telles remarques soient délibérément émises en leur présence.

Dans l'ensemble, les remarques inappropriées relativement au genre sont aussi fréquentes que les propos homophobes faisant référence à des orientations non hétérosexuelles, constatent les auteur·e's. Dans leur présentation des résultats 
d'enquête, que ce soit dans cette section ou ailleurs dans le rapport, les difficultés rencontrées par les élèves trans sont soulignées à plusieurs reprises et je ne mets pas en doute ce constat. Toutefois, la discussion des données sur le sexisme est escamotée. Aucun paragraphe n'aborde les répercussions possibles de cette situation sur les filles de minorités sexuelles qui entendent de tels propos sexistes. Sur le plan conceptuel, un glissement s'opère entre commentaires négatifs sur les filles/garçons (ou sexisme) et commentaires transphobes (sur la non-conformité de genre), qui sont assimilés les uns aux autres dans l'expression plus globale de « commentaires négatifs reliés au genre ou transphobes" (negative-gender related or transphobic comments). Rappelons que le terme homophobie englobe ici les violences verbales lesbophobes puisque les commentaires qualifiés d'homophobes incluent les insultes dénigrant les lesbiennes ou le lesbianisme. En somme, l'identité de genre est nommée et sert de catégorie d'analyse des résultats, mais l'analyse du sexisme est évacuée et le procédé classificatoire qui fusionne dans un même ensemble commentaires sexistes et commentaires transphobes obscurcit plutôt qu'il n'éclaire les enchevêtrements entre sexisme, homophobie et transphobie.

\section{La lesbophobie : une réalité secondarisée}

Une autre section du rapport présente des données détaillées sur la victimation directe, soit le harcèlement verbal et le harcèlement physique attribués par les répondant·e $\cdot s$ à l'un des quatre motifs suivants: 1) orientation sexuelle; 2) genre; 3) expression de genre (la façon dont vous parlez, marchez, vous habillez) ; 4) orientation sexuelle aperçue [définie dans une section introductive comme "lorsqu'une personne pense que vous êtes lesbienne, gaie ou bisexuelle sans connaître votre orientation sexuelle»] ou identité de genre ${ }^{5}$. Le questionnaire

\footnotetext{
${ }^{5}$ Les définitions sont tirées de la version française du questionnaire d'enquête. Il y aurait lieu ici d'approfondir la critique proprement méthodologique d'un tel procédé : comment expliquer le regroupement « orientation sexuelle perçue et identité de genre»? Peut-on postuler que les répondant·e's pourront attribuer le harcèlement subi à tel ou tel motif? Des élèves de 12-13 ans sont-
} 
d'enquête propose également des définitions d'autres termes, dont deux "souvent liés à celui de trangenre" : l'identité de genre est le «sentiment profond d'individuation, de différenciation et d'appartenance à l'un ou l'autre sexe », alors que l'expression de genre est la «façon de présenter son identité de genre en public par rapport à ses vêtements, paroles, langage corporel et d'autres façons de démontrer la masculinité ou la féminité ». Cependant, le terme " genre » n'est jamais luimême défini dans ce rapport d'enquête.

Dans l'ensemble, ces données confirment que les élèves LGBTQ, pris globalement, sont l'objet de harcèlement verbal et physique dans des proportions nettement supérieures à celles observées pour les autres élèves, mais ces dernier-ère's ne sont pas non plus épargné $\cdot e \cdot s$, en particulier en raison des motifs « genre » et « expression de genre ». Ce sont les élèves trans qui écopent le plus, quel que soit le motif de harcèlement considéré. Comparativement aux garçons, les filles lesbiennes, bisexuelles ou en questionnement sont plus nombreuses à rapporter du harcèlement verbal ou physique pour trois des quatre motifs considérés, soit l'orientation sexuelle, réelle et perçue, l'expression de genre et l'identité de genre. À l'inverse, les garçons gais, bisexuels ou en questionnement rapportent davantage de harcèlement qu'ils associent à leur genre, comparativement aux filles (p. 61). Les auteur·e-s s'étonnent de ce résultat, mais n'en proposent pas d'explication. Comment les élèves ont-ils interprété les nuances entre ces sous-questions et les définitions fournies en début de questionnaire? Impossible de le savoir malgré les commentaires libres ajoutés par certain $\cdot e \cdot s$ répondant $\cdot e \cdot s$.

Sur la base de ces résultats ainsi que d'autres données concernant le harcèlement sexuel, la propagation de rumeurs et le sentiment de sécurité à l'école, le rapport réfute l'idée commune selon laquelle, comparativement aux jeunes gais et bisexuels, les filles lesbiennes et bisexuelles seraient plus facilement tolérées dans le milieu scolaire, voire qu'elles seraient considérées comme à la mode (trendy). En revanche,

ils capables de saisir les nombreuses définitions présentées au début du questionnaire? 
dans l'ensemble de l'enquête et du rapport qui en a découlé, les violences de genre sont conceptualisées en termes d'identité de genre et d'expression de genre, soit des expériences subjectives $\mathrm{du}$ genre. Les élèves trans, conclut le rapport, sont les principales victimes de la régulation rigide des genres: « ielles » sont des cibles fréquentes du harcèlement entre pairs, tantôt en raison de leur expression de genre non conforme aux

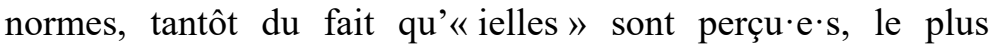
souvent erronément, comme gais, lesbiennes ou bisexuel·le·s.

Par ailleurs, le caractère systémique de ces discriminations est souligné en recourant aux termes d'homophobie, de biphobie et de transphobie dont la culture scolaire est imprégnée. Le terme «lesbophobie» n'est employé qu'une seule fois dans le rapport, alors que celui de transphobie fait l'objet de 36 mentions, incluant l'expression anti-transphobie. Selon les recommandations $\mathrm{du}$ rapport, les politiques antidiscrimination doivent inclure explicitement l'homophobie, mais également proposer des mesures concernant l'identité de genre, l'expression de genre et la transphobie. En revanche, le sexisme n'est pas spécifiquement mentionné dans les recommandations. Enfin, l'étude ne s'est pas intéressée aux auteur.e's des violences, de quelque nature que ce soit. Les résultats de l'unique question sur l'émission de commentaires homophobes ne sont pas inclus dans le rapport d'enquête.

\section{Étude 2 - L'homophobie à l'école secondaire au Québec : une imbrication partielle des violences homophobes et des violences liées au genre}

Le rapport L'homophobie à l'école secondaire au Québec (Chamberland et al., 2011) brosse un portrait du climat scolaire relativement à l'homophobie dans les établissements scolaires québécois offrant de l'enseignement aux élèves de 14 à 17 ans et en examine les impacts sur la persévérance et la réussite scolaires ${ }^{6}$. Certains choix méthodologiques ont été influencés

\footnotetext{
${ }^{6}$ Les autres volets de cette recherche ne seront pas considérés ici, soit l'étude qualitative auprès des jeunes trans, l'enquête auprès des élèves fréquentant les cégeps (17 ans et plus), les entretiens avec des élèves sur les dimensions
} 
par des constats ressortant de la recension de la littérature scientifique. Ainsi l'enquête s'adressait à tous les élèves québécois, sur la base d'un échantillon représentatif de la population fréquentant les écoles secondaires à travers le Québec. En effet, une conclusion ressortait de quelques études s'appuyant sur de très grands échantillons incluant des élèves de toutes orientations sexuelles : si les élèves LGB risquaient plus que leurs pairs exclusivement hétérosexuels de subir diverses formes d'agression, les gestes et propos homophobes ciblaient également des élèves présumés l'être sur la base de comportements, de styles vestimentaires, de choix de filières, de préférences sportives ou ludiques non conformes aux stéréotypes de genre. Le questionnaire s'est inspiré des études sur l'intimidation en général, afin d'établir une liste d'items ${ }^{7}$ permettant de cerner toutes les manifestations possibles de l'intimidation à caractère homophobe. Pour chacun des items, il était demandé si l'élève avait déjà été victime de tels agissements, et à quelle fréquence, parce qu'il ou elle était gai, lesbienne ou bisexuel·le ou parce qu'on pensait qu'il ou elle l'était. Les mêmes items étaient repris pour interroger les répondant $\cdot \mathrm{e} \cdot \mathrm{s}$ sur des situations et des gestes dont ils ou elles avaient pu avoir été témoins (directs ou indirects) ou auteur·e $\mathrm{s}$.

\section{L'homophobie comme sanction de la non-conformité de genre}

Les résultats obtenus indiquent que: 1) les élèves s'identifiant comme hétérosexuel-le-s sont très nombreux $(35,4 \%)$ à se déclarer victimes d'au moins un incident à caractère homophobe pendant l'année scolaire en cours, que l'on considère chaque type d'incident ou que l'on retienne le résultat global, indistinctement du type d'incident ; 2) les élèves s'identifiant comme LGBQ sont toujours significativement plus nombreux à rapporter de tels incidents, quel que soit le type

psychosociales des expériences de victimation en lien avec la persévérance et la réussite scolaires, et la recension des outils et guides d'intervention disponibles. La recherche a été financée par le Fonds québécois de recherche Société et Culture (FQRSC).

${ }^{7}$ Voici deux exemples d'items : 1) Se faire bousculer, frapper, donner des coups de pied, se faire cracher dessus ou lancer des objets 2) Se faire insulter, taquiner méchamment, subir des moqueries, se faire humilier. 
d'incident ainsi que globalement, avec un total de $69 \%$ d'entre eux qui en déclarent au moins un (p. 14). D'autres résultats, calculés à l'aide d'un score de victimation créé afin de prendre en considération la fréquence des incidents subis, montrent qu'une proportion plus grande d'élèves LGBQ rapportent vivre des incidents de manière répétée ou intense, comparativement aux élèves auto-identifiés comme hétérosexuel·e·s (Chamberland, Richard et Bernier, 2013). Cela dit, en nombre absolu, les élèves hétérosexuels, largement majoritaires, sont plus nombreux à être la cible d'incidents à caractère homophobe.

Une autre question mesurait les perceptions des répondant $\cdot \mathrm{e} \cdot \mathrm{s}$ concernant les motifs pour lesquels des jeunes sont susceptibles d'être taquinés, insultés ou intimidés dans leur environnement scolaire. Sur les huit motifs proposés, les trois qui se sont hissés aux premiers rangs, avec une avance importante, sont : 1) l'apparence, la taille, la forme du corps et le poids ; 2) parce qu'un garçon se comporte de manière trop féminine ou une fille de manière trop masculine ; 3) parce qu'ils et elles sont ou que l'on pense qu'ils et elles sont gais, lesbiennes ou bisexuel-le·s. Le motif "sexe» arrive en tout dernier, après des motifs liés au handicap, à la religion et à l'origine ethnoculturelle.

Comment interpréter ces résultats au-delà de la description fournie dans le rapport d'enquête? Tout d'abord, la nonconformité aux normes de genre est sanctionnée en tant que telle par les pairs, tout comme l'écart aux normes corporelles. Ensuite, une apparence ou une conduite jugée non conforme aux normes de genre serait perçue par certains pairs comme un indice qui fait naître ou confirme des soupçons d'homosexualité - déclenchant alors des manifestations d'hostilité et de rejet. Autrement dit, les anciens stéréotypes associant l'homosexualité à l'inversion de genre demeureraient vivaces dans les jeunes générations. Ou encore, tout en ne questionnant pas l'hétérosexualité d'un ou d'une élève, des pairs lui accoleraient une étiquette stigmatisante (fif, gouine, etc.) afin de sanctionner son incapacité à démontrer suffisamment sa masculinité ou sa féminité. 
Cette dernière interprétation est confortée par l'étude de Janik Bastien Charlebois (2011a) sur les discours homophobes des garçons adolescents, notamment l'emploi péjoratif de termes tels que gai, fif ou tapette adressés le plus souvent par des garçons à d'autres garçons. Ceux-ci se défendent bien de cibler précisément des gais ou de se méprendre sur l'orientation sexuelle d'un pair lorsqu'ils émaillent leurs propos d'épithètes puisées dans la gamme des termes dénigrant l'homosexualité. En revanche, selon l'analyse de Bastien Charlebois (2011a), l'usage de ces épithètes relève du sexisme: la dévalorisation d'un pair ou d'une conduite qui ne satisfait pas aux exigences de la virilité s'opère à travers son association à l'efféminement et donc ultimement au féminin, caractérisé par la faiblesse, l'incapacité, la peur, bref, doté d'une valeur moindre.

Ce rapport d'enquête confirme la nécessité de se pencher non seulement sur le vécu des jeunes de minorités sexuelles, mais également sur les expériences des élèves hétérosexuels, garçons et filles, qui peuvent être vulnérables à la victimation revêtant un caractère homophobe. Sur le plan théorique, l'imbrication entre violences homophobes et violences liées au genre est alors conçue comme une même réponse négative, voire agressive, que l'on qualifiera d'homophobe, face à l'homosexualité et face à la dérogation aux modèles de féminité et de masculinité, ou ce que certains appellent «l'expression de genre». Selon cette ligne d'analyse sociologique (Lerch et Chauvin, 2013), l'homophobie ne sanctionne pas tant les rapports sexuels entre personnes de même sexe que l'affichage de traits traditionnellement attachés à l'autre sexe. Elle se rapproche alors de la transphobie et de la stigmatisation des déviances de genre.

Tant la recherche que les programmes d'intervention devraient donc s'adresser à l'ensemble des jeunes, d'autant que nombre d'ados se disent indécis ou en questionnement quant à leur orientation sexuelle ou présentent des " dissonances » entre leurs attirances, leurs comportements sexuels et leur autoidentification. Les études récentes basées sur de larges échantillons tendent d'ailleurs à différencier plus finement entre eux les sous-groupes de minorités sexuelles, et mettent à jour la vulnérabilité des élèves en questionnement, bisexuel·le·s ou 
principalement mais non exclusivement hétérosexuel·le·s (voir entre autres Cénat et al., 2015 ; Saewyc et al., 2014). Il est donc souhaitable que les recherches futures sur l'intimidation ou sur la santé des adolescent $\cdot e \cdot s$ incorporent des mesures de l'orientation sexuelle tenant compte de ses dimensions d'attirances, de comportements, et d'identités (Toomey et Russell, 2016 ; Beaulieu-Prévost et Fortin, 2015).

\section{Un éclairage trop partiel des violences interpersonnelles subies par les filles}

Une autre façon d'introduire le sexe et le genre dans l'étude des manifestations d'homophobie est d'examiner la différence de positionnement des garçons et des filles comme victimes mais aussi comme auteur.e's de propos et de gestes violents. Conformément à la politique gouvernementale du Québec, cette étude devait inclure une analyse différenciée selon le sexe (ADS) en comparant garçons et filles. Les résultats ne corroborent pas la conception commune selon laquelle seuls quelques garçons fauteurs de trouble poseraient des gestes d'intimidation à caractère homophobe. Également, tout comme l'étude de Taylor et al. (2011) précédemment examinée, ils contredisent la croyance, souvent véhiculée dans le milieu associatif LGBTQ, que seuls les jeunes gais seraient victimes d'homophobie, alors que les jeunes lesbiennes se tireraient facilement d'affaire à l'école.

Dans l'ensemble, les données révèlent des dynamiques auxquelles participent garçons et filles sans que les uns ou les autres aient le monopole d'une forme de violence, exercée ou subie, et la prudence s'impose dans l'interprétation des données. Les garçons sont plus nombreux que les filles à entendre souvent des remarques et des insultes homophobes et font plus souvent l'objet de bousculades et de coups, ainsi que d'insultes, taquineries méchantes et humiliations. Pour leur part, les filles sont plus sujettes à la victimation de nature sexuelle - notamment les avances hétérosexuelles insistantes, les gestes sexuels posés contre leur gré - et à la cyberintimidation (Chamberland et al., 2010). Concernant l'exercice de la violence, une proportion plus grande de garçons que de filles déclare poser des gestes à caractère homophobe pour la majorité 
des items proposés dans l'enquête, à l'exception des rumeurs lancées dans le but de nuire à la réputation et de la cyberintimidation dans laquelle on n'observe aucune différence significative. Enfin, les garçons obtiennent des scores plus élevés que les filles sur le plan des attitudes négatives envers l'homosexualité.

La principale limite d'un tel portrait de l'intimidation à caractère homophobe est de cerner les gestes de violence rapportés par les élèves comme étant motivés ou associés à un rejet de l'homosexualité ou de la bisexualité, réelle ou perçue, en recourant à la formule " parce qu'on pensait que vous étiez gai, lesbienne ou bisexuel·le...». D'une part, il s'avère quelque peu hasardeux de présumer de l'interprétation donnée par les répondant·e-s à une telle formulation. D'autre part, les données recueillies demeurent lacunaires en ce qui concerne la situation respective des garçons et des filles quant à l'intimidation en lien avec leur sexualité. Ainsi, on peut penser que les filles de toutes orientations sexuelles sont la cible d'insultes sexuelles qui ne s'inscrivent pas dans le registre de l'homophobie ou de la lesbophobie (par exemple bitch, pute, garce. Sur le slut-shaming ou le stigmate de la pute, voir Tanenbaum, 2015 et Clair, 2012), sans compter les commentaires sexistes ainsi que les conduites de harcèlement et de violence, y compris de violence sexuelle, qui ne sont pas associées, aux yeux des répondantes, à leur orientation sexuelle non hétérosexuelle (réelle ou perçue). La comparaison entre garçons et filles n'éclaire donc que partiellement leur situation respective quant aux violences subies en lien avec leur genre et leur sexualité. Enfin, sauf exception, la question du sexe et du genre des auteur'e.s d'actes de violence, qui permettrait de cerner les dynamiques des violences interpersonnelles garçons/filles, n'est généralement pas enquêtée dans les études sur l'intimidation. Si elle l'est, comme dans le cas de cette enquête, elle n'est guère discutée dans les publications scientifiques et grand public, ce qui limite le portrait global que l'on pourrait dresser des violences de genre parmi les adolescent.e.s. 


\section{Étude 3 - The 2013 National School Climate Survey : un affinement des motifs de victimation, mais une négligence du sexisme}

Comme les études précédentes, le National School Climate Survey de GLSEN s'intéresse au climat scolaire sous l'aspect du langage LGBT-phobe (commentaires négatifs, insultes) et des expériences de victimation (harcèlement verbal et physique, agressions physiques). L'édition de 2013 a rejoint 7898 élèves de 13 à 21 ans, provenant de tous les États. Le questionnaire d'enquête de même que les modes de recrutement des répondant $\cdot \mathrm{e} \cdot \mathrm{s}$ ont évolué depuis la mise en place de cette enquête bisannuelle au tournant des années 2000. Les répondant $\cdot e \cdot s$ doivent satisfaire aux critères suivants : s'autoidentifier comme lesbienne, gai, bisexuel-le ou d'une orientation sexuelle autre qu'hétérosexuel·le (par exemple queer, en questionnement) ou comme transgenre ou ayant une identité de genre autre que cisgenre. Ils et elles sont recrutés à travers des réseaux locaux, régionaux et nationaux d'organismes offrant des services ou œuvrant à la défense des droits des jeunes de minorités sexuelles et de genre, ainsi que, de plus en plus, à travers les réseaux sociaux virtuels (comme Facebook). L'échantillon, non représentatif, se compose de volontaires ; au fil des ans, des efforts ont été déployés pour l'étendre sur le plan géographique (par exemple dans des communautés rurales), le diversifier dans sa composition ethnoculturelle (par exemple jeunes Noir·e-s ou racisé.e.s) ainsi que pour augmenter la participation des jeunes trans. Tous les répondant $\cdot e \cdot s$ sont invité $e \cdot s$ à identifier leur orientation sexuelle et leur identité de genre. Les termes d'autodésignation montrent une tendance au rejet des identités binaires ou exclusives. Ainsi moins de $60 \%$ des répondant·e's s'identifient comme gais ou lesbiennes alors que près du tiers $(31,6 \%)$ se disent bisexuel·le·s/pansexuel·le's et 4,8 \% queer.

Pour ce qui est de l'identité de genre, le quart des répondant $\cdot \mathrm{e} \cdot \mathrm{s}$ se revendiquent d'une identité autre que homme (male) ou femme (female): transgenre, genderqueer ou d'un autre genre (agender, pangender...). Pour ce qui est de l'expression de genre, il est demandé aux répondant $\cdot e \cdot s$ de 
qualifier la perception de leur apparence ou de leur comportement par leur entourage scolaire sur une échelle de sept degrés, allant de "très masculin » à «très féminin » en passant par «également masculin et féminin ». Cette question permet ensuite de classer les répondant $\cdot e \cdot s$ selon leur conformité ou leur non-conformité aux normes dominantes d'expression de genre (gender nonconformity).

Contrairement aux deux études précédentes, l'enquête de GLSEN est en mesure de présenter des résultats qui comparent la victimation subie par des élèves de diverses orientations sexuelles et de diverses identités de genre, à l'exception des élèves à la fois exclusivement hétérosexuel·le $\cdot \mathrm{s}$ et cisgenres qui sont absent·e.s de l'échantillon, et de le faire sans les agglomérer dans un même ensemble, étant donné la grandeur et la diversité interne de l'échantillon de jeunes répondant·e $\cdot s$. De plus, le questionnaire d'enquête distingue les violences fondées sur l'orientation sexuelle de celles fondées sur la nonconformité de genre.

\section{Le langage sexiste : constaté, mais non analysé}

Le rapport de l'enquête 2013 (Kosciw et al., 2014) trace d'abord un portrait global des violences en contexte scolaire fondées sur l'orientation sexuelle et sur l'expression de genre telles que rapportées par l'ensemble des répondant LGBTQ. Une première section est consacrée à l'exposition à divers biais de langage en contexte scolaire. Une large majorité (de $56 \%$ à $74 \%$ ) déclare entendre souvent ou fréquemment des commentaires homophobes (It's so gay), des termes dénigrants tels que fag, dyke ou des remarques négatives à propos de l'expression de genre, c'est-à-dire jugeant défavorablement un·e élève qui n'agit pas de manière jugée assez masculine ou assez féminine (p. 16-18).

Si l'on compare l'ensemble des types de langage biaisé, on constate que les remarques sexistes occupent le premier rang, toutes catégories confondues, alors que la fréquence des propos dénigrants relatifs aux capacités, au poids ou à la grosseur se compare à celle des propos concernant l'orientation sexuelle et l'expression de genre, et que les propos racistes ou négatifs envers la religion sont relativement moins fréquents. Le rapport 
présente l'ensemble de ces biais langagiers sans toutefois s'attarder à discuter des impacts des propos sexistes sur les filles (cisgenres ou trans) ou sur leurs implications en termes d'inégalités sociales de sexe.

\section{La victimation des filles en lien avec le genre : constatée mais non analysée}

Sur le plan des violences subies, l'orientation sexuelle et l'expression de genre ressortent comme les deux caractéristiques personnelles les plus fréquemment associées à une forme ou l'autre de victimation, suivies du genre, de la race ou l'ethnicité et du handicap. Les données confirment l'ampleur $\mathrm{du}$ harcèlement verbal en lien avec l'orientation sexuelle (près des trois quarts des répondant·e $\cdot s$ ) et avec l'expression de genre (plus de la moitié), ainsi que celle du harcèlement physique (un répondant $\cdot e$ sur trois en lien avec l'orientation sexuelle et plus d'un sur cinq en lien avec l'expression de genre). La très grande majorité des répondant'e-s déclare aussi de l'exclusion sociale et des rumeurs ou mensonges lancés sur leur compte, et de $43 \%$ à $59 \%$ du harcèlement sexuel, de la cyberintimidation ou du vol ou dommage à leurs biens.

Dans une autre section (p. 84-90), le rapport propose une comparaison détaillée et systématique du sentiment de sécurité à l'école et des expériences de victimation verbale ou physique selon les caractéristiques personnelles des répondant·e·s. En référence au genre, deux constats ressortent nettement. Premièrement, les élèves transgenres sont davantage susceptibles de ressentir un sentiment d'insécurité à l'école et d'expérimenter un climat scolaire hostile mis en évidence par les divers indicateurs de victimation fondée sur l'expression de genre et sur le genre. Notons que, comme dans d'autres études (Taylor et Tracey, 2011; Blais et al., 2013), ielles sont également les plus ciblés par la victimation fondée sur l'orientation sexuelle bien que, de fait, leur orientation sexuelle ne soit pas toujours non hétérosexuelle. Les répondant·e·s s'auto-identifiant comme genderqueer ou d'un " genre autre » rencontrent eux aussi un degré d'hostilité plus élevé que ceux s'auto-identifiant dans les cases traditionnelles de sexe et de genre (ou cisgenres). 
Deuxièmement, parmi les répondant $\cdot e \cdot s$ cisgenres, les garçons rapportent des expériences plus négatives que les filles relativement à l'orientation sexuelle et à l'expression de genre, et les filles rapportent des expériences plus négatives que les garçons relativement au genre, notamment s'agissant de harcèlement verbal et physique. Concernant le premier point, les auteur'e's reprennent l'explication selon laquelle notre société accorde plus de latitude ou de fluidité aux filles qu'aux garçons quant à l'orientation sexuelle et à l'expression de genre - l'exemple classique étant celui du port de vêtements ou l'adoption de comportements dits masculins par une fille comparativement à des vêtements ou des conduites féminines pour un garçon. Concernant le second point, à part le qualifier de « sexiste» (p. 88), aucun commentaire n'est formulé pour expliquer le supplément de victimation potentiellement subie par les filles LGB en lien avec leur sexe, soit du fait qu'elles sont des filles.

La répartition des réponses est similaire pour ce qui est du sentiment de sécurité à l'école et les expériences de victimation en lien avec la non-conformité de genre, c'est-à-dire que l'on constate les niveaux les plus élevés d'insécurité et de victimation chez les répondant·e's non cisgenres. Parmi les répondant $\cdot \mathrm{e} \cdot \mathrm{s}$ cisgenres, la non-conformité de genre accroît le sentiment d'insécurité à l'école et les risques de victimation, tant en lien avec l'orientation sexuelle qu'avec l'expression de genre. Le chapitre se conclut en soulignant la nécessité de s'attaquer non seulement à l'homophobie, mais également à la transphobie, au racisme, au sexisme et à toutes les autres formes de biais affectant tous les élèves (p. 90).

En somme, l'édition 2013 de l'enquête GLSEN inclut des questions particulières sur l'orientation sexuelle, l'identité de genre et l'expression de genre des répondant $\cdot e \cdot s$, ce qui permet des comparaisons plus fines selon leur auto-identification relativement à ces trois dimensions. L'enquête collecte également des informations distinctes sur les violences attribuées par les répondant $\cdot e \cdot s$ à l'orientation sexuelle, à l'expression de genre et au positionnement de genre. Cependant, ce dernier motif n'est jamais approfondi. Dans l'ensemble, l'accent est mis sur les variances d'identité et 
d'expression de genre, traitées d'une manière affinée, et les violences ciblant les filles en raison de leur sexe ou ciblant le « féminin » ne sont guère discutées.

\section{Les violences LGBT-phobes entre élèves : un révélateur partiel des violences de genre}

Les trois études brièvement examinées dans cet article démontrent l'ampleur des violences LGBT-phobes sous différentes formes : harcèlement verbal et physique, agressions physiques, exclusion sociale et violences sexuelles. À travers divers éclairages, elles montrent que les violences découlant de l'attribution à un ou une élève d'une orientation sexuelle autre qu'hétérosexuelle, qu'elle soit exacte ou erronée, et les violences associées aux rapports sociaux de sexe ou à la nonconformité de genre s'enchevêtrent et que l'on ne saurait examiner les unes en omettant les autres.

Portant sur des populations mixtes, les deux premières enquêtes font également ressortir que tous les élèves courent le risque d'être l'objet de violences à caractère homophobe ou transphobe, bien que les élèves LGBTQ soient plus fréquemment et plus intensément victimisés - des résultats que confirment des métasynthèses (Toomey et Russell, 2016; Fedewa et Ahn, 2011). La non-conformité aux normes de genre, quelle que soit l'orientation sexuelle, est sanctionnée par les pairs en tant que telle. Ainsi, les élèves hétérosexuel·le·s peuvent devenir la cible de propos et de gestes à caractère homophobe lorsqu'ils ou elles dérogent aux modèles normatifs de genre et se voient imputer une sexualité disqualifiée, que ce soit pour discréditer un comportement jugé inapproprié, car considéré comme trop féminin/masculin, ou parce que cet écart à la norme de genre est vu comme le signe d'une hétérosexualité non exclusive. Selon le rapport de GLSEN (Kosciw et al., 2014), les élèves trans forment le groupe le plus exposé à la victimation, que ce soit en raison de leur identité de genre, de leur non-conformité de genre ou de leur orientation sexuelle perçue. En définitive, les violences LGBT-phobes 
punissent la non-conformité aux attentes hétéronormatives relatives au genre et à la sexualité (voir aussi Blais et al., 2013).

Cependant, tout en montrant que les violences interpersonnelles liées à la sexualité et au genre ne peuvent être dissociées, ces trois études ne permettent pas d'analyser en profondeur, que ce soit sur le plan empirique ou conceptuel, les dynamiques interactionnelles entre pairs, où se conjuguent la pression à la conformité aux normes de genre et l'imposition d'une norme hétérosexuelle, de même que les inégalités et les rapports de pouvoir qui les traversent. Du fait que leur approche est principalement quantitative et qu'elles mettent l'accent sur la victimation, elles butent sur des limites épistémologiques.

Ainsi, malgré l'insertion de remarques formulées par quelques répondant $\cdot e \cdot s$, trop peu d'éléments sont fournis pour qu'il soit possible de déchiffrer les significations accordées aux divers motifs d'exercice des violences (orientation sexuelle, non-conformité de genre, genre) par les répondant·e $\cdot s$ qui disent en être la cible ou les témoins (voir aussi Toomey et Russell, 2016). Bien plus, les joutes d'insultes entre élèves revêtent des significations différentes « selon le contexte de leur énonciation et le contexte relationnel des protagonistes » (Durif-Varembont et Weber, 2014, p. 154), comme le révèlent des travaux de nature ethnograhique reposant sur l'observation de telles dynamiques au quotidien. Au-delà du décryptage des motifs attribués aux violences rapportées, l'angle retenu - celui de la victimation autorapportée - n'éclaire que partiellement le rôle des insultes sexistes et homophobes dans la socialisation identitaire genrée.

Plus particulièrement, les violences attribuées au sexe et au genre, qui ciblent davantage les filles (Taylor et Peter, 2011 ; Kosciw et al., 2014) ou certaines formes de violences qui touchent davantage ces dernières, comme les violences sexuelles (Chamberland et al., 2011), apparaissent au second plan. En d'autres termes, le point de mire de ces études ne permet pas de considérer l'ensemble des violences exercées spécifiquement sur les filles, notamment dans le registre des manifestations du sexisme, de la contrainte normative à l'hétérosexualité pour toutes et de l'imposition d'un double 
standard sexuel où la figure de la «pute » sert de modèle repoussoir (Clair, 2012; Tanenbaum, 2015). Quant à la question des auteur'e.s des violences interpersonnelles (qui exerce de la violence envers qui et sous quelles formes?), elle est omise dans deux des trois études examinée ${ }^{8}$. On peut donc leur reprocher de négliger les positionnements respectifs des garçons et des filles dans l'exercice de ces violences interpersonnelles et les inégalités ainsi reproduites.

Enfin, dans ces études, la notion de genre est considérée sous l'angle individuel des identités et des expressions de genre, et de leurs variations. L'asymétrie entre filles et garçons, entre inculcation de la féminité et de la masculinité - et l'apprentissage plus ou moins forcé de l'hétéronorme dans la construction de cette féminité/masculinité (pour des exemples, voir Lebreton, 2017 ; Clair, 2012 ; Pascoe, 2011) - n'est pas véritablement réfléchie, théorisée, sans compter la complexité d'une articulation avec d'autres rapports sociaux qui obligerait à parler de féminités/masculinités (au pluriel). À ce propos, signalons qu'aucune des trois études ne s'intéresse à la classe sociale et que seule l'étude de GLSEN prend en compte la « race» en l'envisageant comme un attribut individuel et un motif possible de harcèlement.

Sauf pour la recherche de Chamberland et al. ${ }^{9}$, c'est plutôt la notion de transphobie qui est convoquée pour rendre compte du caractère systémique des violences sanctionnant les identités et les conduites qui dérogent ou embrouillent les normes de genre. Or tout comme l'homophobie (Chamberland et Lebreton, 2012; Bastien Charlebois, 2011b), la transphobie est un concept puissant sur le plan pédagogique, car facilement compréhensible, mais faible sur le plan de l'explication sociologique, car réduisant les causes à une question d'attitudes

\footnotetext{
8 De son côté, la recherche de Chamberland et al. fournissait quelques données à ce propos, mais celles-ci n'ont guère été exploitées dans la diffusion auprès du grand public.

${ }^{9}$ Dans cette recherche, l'emploi du terme de transphobie a été restreint aux violences rapportées par les élèves trans, qui ont fait l'objet d'un rapport d'enquête distinct. Ma critique de l'extension théorique donnée à concept s'applique donc aux deux autres rapports d'enquête.
} 
individuelles. La conceptualisation des observations rapportées à travers la loupe de la transphobie permet d'insister sur la stigmatisation des personnes qui tentent d'échapper à l'imposition des modèles hétéronormatifs. Elle est sans doute à mettre en relation avec l'influence de la théorie queer et du concept d'hétéronormativité (Warner, 1999) où l'accent est mis sur la normativité s'exerçant à travers la binarité des catégories du système de sexe et de genre. La notion de transphobie vient donc effacer une autre vision du genre comme un système de bicatégorisation hiérarchisée entre les sexes et entre les valeurs et les représentations qui leur sont associées, en d'autres termes une vision du genre comme rapport social (re)producteur des inégalités entre hommes et femmes (Couchot-Schiex, Moignard et Richard, 2016 ; Bereni et al., 2012).

Par ailleurs, le concept de lesbophobie est mis de côté. Or ce concept permettrait de relier l'ensemble des violences verbales, psychologiques, physiques ou sexuelles ciblant les lesbiennes ou le lesbianisme, d'en révéler la variété et les degrés divers de gravité, et d'en dénoncer plus clairement les impacts individuels et sociaux sur les filles et les femmes de toutes orientations sexuelles. Certes, ce concept présente aussi des limites : tout comme l'homophobie ou la transphobie, il est plus apte à décrire des attitudes et des comportements individuels que des processus sociaux, dont l'explication renvoie le plus souvent à des déterminants eux aussi individuels. On lui a également reproché de dépolitiser l'existence lesbienne et de réduire le lesbianisme à une simple préférence sexuelle, plutôt qu'une remise en cause de l'hétéropatriarcat (Kitzinger et Perkins, 1993 ; Crawford, 2012).

Cependant, malgré ces limites, son usage inviterait à conjuguer l'examen empirique des violences fondées sur l'orientation sexuelle et des violences fondées sur l'appartenance (ou l'assignation) à la catégorie du sexe féminin, que ces violences soient exercées sur les filles LGBQ ou sur les filles en général. Une telle conjugaison apparaît préférable à ma mise de côté des observations sur les remarques sexistes et les violences vécues par les filles en lien avec leur genre ou du fait qu'elles sont des filles, comme c'est le cas des études précédemment examinées. Pour le dire autrement l'emploi du 
concept de lesbophobie exigerait d'explorer l'intersection entre genre et homophobie, c'est-à-dire d'approfondir les dissemblances entre les processus de disqualification de l'homosexualité en fonction du sexe et du genre (Arc et Vellozo, 2012 ; Chamberland et Lebreton, 2012 ; Gérard, 2003 ; Worthen, 2013).

En somme, si les recherches présentées ici dévoilent certains mécanismes d'imbrication du sexisme et de l'homophobie, elles laissent dans l'ombre l'analyse de l'ensemble des processus d'inculcation de l'hétérosexualité aux filles et tendent à diluer l'analyse des rapports sociaux de sexe en l'envisageant uniquement sous l'angle de la non-conformité aux normes de genre, ou de dérogation à l'impératif d'hétéronormativité, tout en niant leur asymétrie intrinsèque.

\section{Remerciements}

Je remercie l'équipe éditrice de même que les personnes ayant évalué une première version de cet article pour leurs commentaires avisés.

\section{Références}

Arc Stéphanie, et Vellozzo Phillipe (2012). «Rendre visible la lesbophobie ». Nouvelles questions féministes, 31 (1), p. 12-26.

Aultman B. (2014). "Cisgender». TSQ: Transgender Studies Quaterly, 1 (1-2), p. 61-62.

Bastien Charlebois Janik (2011a). La virilité en jeu. Perception de l'homosexualité masculine par les garçons adolescents. Montréal, Septentrion.

Bastien Charlebois Janik (2011b). « Au-delà de la phobie de l'homo : quand le concept d'homophobie porte ombrage à la lutte contre l'hétérosexisme et l'hétéronormativité ». Reflets : revue d'intervention sociale et communautaire, 17 (1), p. 112-149.

Beaulieu-Prévost Dominique, Fortin Mélissa (2015). « La mesure de l'orientation sexuelle: historique et pratiques actuelles ». Sexologies, 24 (1), p. 29-34. 
Bereni Laure, Chauvin Sébastien, Jaunait Alexandre, Revillard Anne (2012). Introduction aux études sur le genre, $2^{\mathrm{e}}$ édition revue et augmentée. Bruxelles, De Boeck.

Blais Martin, Gervais Jesse, Boucher Kathleen, Hébert Martine, Lavoie Francine et l'équipe de recherche PAJ (2013). « Prevalence of prejudice based on sexual minority status among 14 to 22-yearold youths in the province of Quebec (Canada)». International Journal of Victimology, 11 (2).

Cénat Jude-Mary, Martin Blais Martin, Martine Hébert Martine, Lavoie Francine, Guerrier Mireille (2015). « Correlates of bullying in Quebec high school students: The vulnerability of sexualminority youth ». Journal of Affective Disorders, 183, p. 315-321.

Chamberland Line, Émond Gilbert, Bernier Michaël, Richard Gabrielle, Petit Marie-Pier et al. (2011). L'homophobie à l'école secondaire au Québec. Portrait de la situation, impacts et pistes de solution. Rapport de recherche, Montréal, Université du Québec à Montréal. http://chairehomophobie.uqam.ca/partage-dessavoirs/publications/rapports-de-recherche/224-1-homophobie-a-1ecole-secondaire-au-quebec-portrait-de-la-situation-impacts-etpistes-de-solution.html

Chamberland Line, Baril Alexandre, Duchesne Natalie (2011). La transphobie en milieu scolaire au Québec. Rapport de recherche. Montréal, Université du Québec à Montréal.

Chamberland Line, Émond Gilbert, Julien Danielle, Otis Joanne, Ryan Bill et al. (2010). L'impact de l'homophobie et de la violence homophobe sur la persévérance et la réussite scolaires. Rapports annexes. Montréal, Université du Québec à Montréal.

Chamberland Line, Richard Gabrielle, Bernier Michaël (2013). « Les violences homophobes et leurs impacts sur la persévérance scolaire des adolescents au Québec ». Recherches \& Éducations, 8, p. 99-114.

Chamberland, Line et Christelle Lebreton (2012). « Réflexions autour de la notion d'homophobie : succès politique, malaises conceptuels et application empirique », Nouvelles questions féministes, vol. 31, $\mathrm{n}^{\circ} 1,27-43$.

Clair Isabelle (2012). «Le pédé, la pute et l'ordre hétérosexuel». Agora débats/jeunesse, 60, p. 67-78.

Couchot-Schiex Sigolène, Moignard Benjamin, Richard Gabrielle (2016). Cybersexisme: une étude sociologique dans des établissements scolaires franciliens. Rapport de l'étude commandée par le Centre Hubertine Auclert, Paris, Centre Hubertine Auclert. 
Crawford Charmaine (2012). "“It's a girl thing." Problematizing female sexuality, gender and lesbophobia in caribbean culture ». Theorizing Homophobias in the Caribbean - Complexities of Place, Desire \& Belonging, Caribbean Region of the International Resource Network disponible sur : http://www.caribbeanhomophobias.org

Dubuc Dominique (2017). LGBTQI2SNBA+. Les mots de la diversité liée au sexe, au genre et à la diversité sexuelle. Montréal, FNEEQCSN.

Durif-Varembont Jean-Pierre, Weber Rebecca (2014). «Insultes en tous genres. Construction identitaire et socialisation des adolescents à l'école ». Nouvelle revue de psychosociologie, 17 (1), p. 151-165.

Fedewa Alicia L., Ahn Soyeon (2011). « The Effects of Bullying and Peer Victimization on Sexual-Minority and Heterosexual Youths: A Quantitative Meta-Analysis of the Literature ». Journal of GLBT Family Studies, 7 (4), p. 398-418.

Gérard Raymonde (2003). "Lesbophobie ». In Louis-Georges Tin (dir.), Dictionnaire de l'homophobie. Paris, PUF, p. 262-264.

Kitzinger Celia, Perkins Rachel (1993). Changing Our Minds : Lesbian Feminism and Psychology. Londres, Only Women Press.

Kosciw Joseph G., Greytak Émily A., Palmer Neal A., Boesen Madelyn J. (2014). The 2013 National School Climate Survey: The Experiences of lesbian, gay, bisexual and transgender youth in our nation's schools. New York, GLSEN.

Lebreton Christelle (2017). Adolescentes lesbiennes en quête de reconnaissance. Montréal, Éditions du Remue-ménage.

Lerch Arnaud, Chauvin Sébastien (2013). La sociologie de l'homosexualité. Paris, La Découverte.

Pascoe Cheri J. (2011). Dude, You're a Fag. Masculinity and Feminity in High School. Berkeley, University of California Press.

Petit Marie-Pier, Chamberland Line, Richard Gabrielle, Marilyne Chevrier (2011). "Jeunes de minorités sexuelles victimes d'homophobie en milieu scolaire : quels facteurs de protection?», Journal of Community Mental Health/Revue canadienne de santé mentale communautaire, 30 (2), p. 13-29.

Saewyc Elizabeth M., Konishi Chiaki, Rose Hilary A., Homma Yuko (2014). «School-based strategies to reduce suicidal ideation, suicide attempts, and discrimination among sexual minority and heterosexual adolescents in western Canada ». International Journal of Child,Youth and Family Studies, 5 (1), p. 89-112. 
Tanenbaum Leora (2015). I Am Not a Slut: Slut-Shaming in the Age of the Internet. New York, Harper Perennial.

Taylor Catherine et Tracey Peter (2011). Every class in every school: The First national climate survey on homophobia, biphobia, and transphobia in Canadian schools. Final report. Toronto, Egale Canada Human Rights Trust.

Toomey Russell B., Russell Stephen T. (2016). « The role of sexual orientation in school-based victimization: A meta-analysis ». Youth \& Society, 48 (2), p. 176-201.

Veale Jaimie, Saewyc Elizabeth, Frohard-Dourlent Hélène, Dobson Sarah, Clark Beth et le groupe de recherche de l'enquête canadienne sur la santé des jeunes trans (2015). Être en sécurité, être soi-même. Résultats de l'enquête canadienne sur la santé des jeunes trans. Vancouver, Stigma and Resilience among Vulnerable Youth Centre, UBC.

Warner, Michael (1999). The Trouble with Normal. Sex, Politics, and the Ethics of Queer Life. New York, Free Press.

Worthen, Meredith G. F. (2013). « An argument for separate analysis of attitudes toward lesbian, gay, bisexual men, bisexual women, MtF and FtM transgender individuals ». Sex Roles, vol. 68, p. 703723. 